\title{
False-positive diagnosis of disease progression by magnetic resonance imaging for response assessment in prostate cancer with bone metastases: A case report and review of the pitfalls of images in the literature
}

\author{
YI-SHAN YU ${ }^{1 *}$, WAN-HU LI ${ }^{2 *}$, MING-HUAN LI ${ }^{1}$, XUE MENG $^{1}$, LI KONG ${ }^{1}$ and JIN-MING YU ${ }^{1}$ \\ ${ }^{1}$ Departments of Radiation Oncology and ${ }^{2}$ Radiology, Shandong Cancer Hospital and Institute, \\ Shandong University, Jinan, Shandong 250117, P.R. China
}

Received November 19, 2014; Accepted August 17, 2015

DOI: $10.3892 / \mathrm{ol} .2015 .3753$

\begin{abstract}
Bone metastases are common in prostate cancer. However, differentiating neoplastic from non-neoplastic alterations of bone on images is challenging. In the present report, a rare case of bone marrow reconversion on magnetic resonance imaging (MRI) assessment, which may lead to a false-positive diagnosis of disease progression of bone metastases in hormone-resistant prostate cancer, is presented. Furthermore, a review of the literature regarding the pitfalls of images for response assessment, including the 'flare' phenomenon on bone scintigraphy, computed tomography (CT), positron emission tomography/CT and marrow reconversion on MRI is also provided. These inaccuracies, which may lead to a premature termination of an efficacious treatment, should be carefully considered by the radiologists and oncologists involved in clinical trials. The case reported in the present study showed how to assess the early therapeutic response and select the appropriate treatment for the patient when these pitfalls are encountered on clinical images.
\end{abstract}

\section{Introduction}

Prostate cancer is the most common malignancy among males worldwide, and is the second leading cause of cancer-related mortality among men in United States (1). Bone metastases occur in $\sim 90 \%$ of the patients presenting with advanced prostate cancer, and there is a direct correlation between the burden of metastases and survival $(2,3)$. Although

Correspondence to: Mr. Jin-Ming Yu, Department of Radiation Oncology, Shandong Cancer Hospital and Institute, Shandong University, 440 Jiyan Road, Jinan, Shandong 250117, P.R. China

E-mail: sdyujinming@126.com

*Contributed equally

Key words: pitfalls of images, prostate cancer, 'flare' phenomenon, marrow reconversion on magnetic resonance imaging numerous effective systematic agents for males with bone metastatic prostate cancer exist, including endocrine therapy for non-hormone-resistant prostate cancer (HRPC) and second-line hormonal therapy and chemotherapy for HRPC and castration-resistant prostate cancer, the clinicians must decide how to sequence these options to maximise their benefit for the patients; what images must be captured prior and post-treatment in order to assess disease status; and how to interpret these images and use them to guide management. However, identifying whether the selected agent is effective for the treatment of prostate cancer with bone metastases is challenging, often due to the uncertainty of interpreting the post-therapy alterations observed on bone scintigraphy (BS) and computed tomography (CT) images as a consequence of the 'flare' phenomenon $(4,5)$. This paradoxical phenomenon refers to an improvement on the levels of prostate-specific antigen (PSA) and pain in patients with bone metastases, which may be accompanied by an initial apparent deterioration of certain lesions or the detection of novel lesions on the images (6).

According to previous reports, MRI has become a promising method to assess the therapeutic response and guide treatment decisions in patients affected by prostate cancer (7). However, the 'flare' phenomenon on MRI has not been reported thus far. In the present report, the first case of a false-positive diagnosis of disease progression on MRI follow-up during systematic therapy of HRPC with bone metastases is described. This case showed that the images captured during the follow-up of patients treated for prostate cancer, which are aimed at assessing the tumor burden and response to therapy, are to be interpreted with caution, in order to avoid a false-positive diagnosis of disease progression and the consequent inappropriate discontinuation of an efficacious therapy. In the present report, the pitfalls of images of bone metastases in patients with prostate cancer were reviewed, including the 'flare' phenomenon on BS and CT, and marrow reconversion on MRI. In addition, potential mechanisms that account for these phenomena were proposed, and subsequent treatment assessment was suggested. Future studies require a more accurate assessment of treatment response in cases of prostate cancer presenting with bone metastases. 

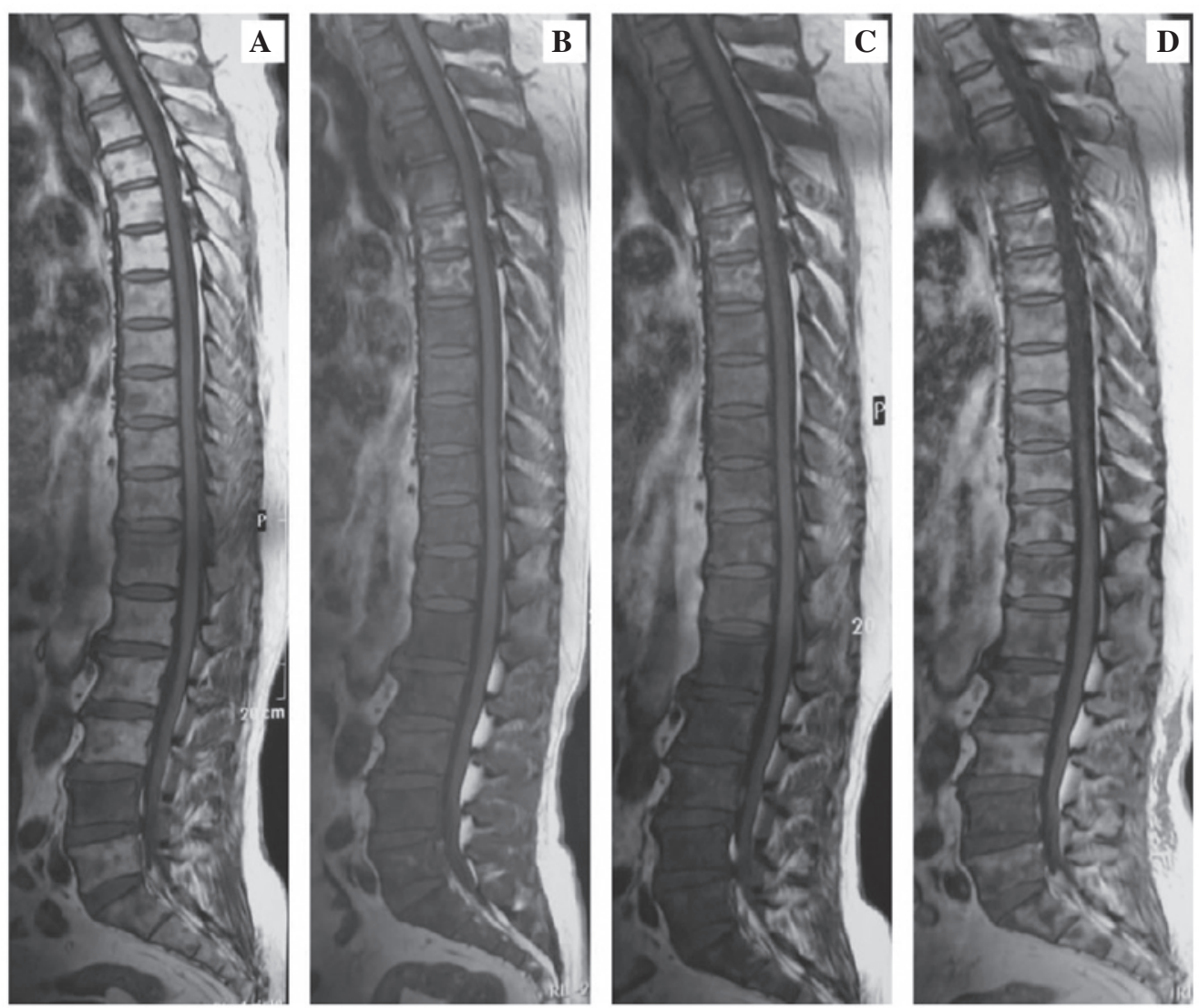

Figure 1. Signal variations observed on MRI T1-weighted images (A) prior to chemotherapy, and following (B) 2, (C) 6 and (D) 10 cycles of chemotherapy treatment. MRI, magnetic resonance imaging.

\section{Case report}

A 67-year-old male, diagnosed in February 2010 with prostate adenocarcinoma with Gleason $9(5+4)$, T4 (infiltration of the posterior urethra), was admitted in June 2011 to the Department of Radiation Oncology of the Shandong Cancer Hospital and Institute at Shandong University (Jinan, Shandong, China) for biochemical progression, following radical prostatectomy. The medical history of the patient included hypercholesterolemia and moderate hypertension treated with betaloc and nifedipine controlled-release tablets (Adalat CC; Bayer China Ltd., Shanghai, China). The levels of PSA in serum at the time of diagnosis were $9.56 \mathrm{ng} / \mathrm{ml}$ (normal range, 0.0-4.0 ng/ml). Following radical prostatectomy, the patient remained asymptomatic with low PSA levels for 15 months (PSA minimum $0.004 \mathrm{ng} / \mathrm{ml}$ ). Since the patient had not received any androgen deprivation therapy, the risk of recurrence was high, and at the time of admission, the patient was diagnosed with biochemical progression and an increased PSA of $13.93 \mathrm{ng} / \mathrm{ml}$. To ascertain the patient's progress, a whole-body positron emission tomography (PET)/CT was conducted that revealed metastases of the T5 vertebral body, which was confirmed by MRI. In consequence, palliative radiotherapy of the metastatic T5 vertebral body and combined androgen blockade therapy were initiated, comprising lutein-releasing hormone analog (Zoladex; AstraZeneca, Shanghai, China) $3.6 \mathrm{mg} / 28$ days and bicalutamide $50 \mathrm{mg} / \mathrm{day}$. Additionally, zoledronic acid was administered intravenously every 3-4 weeks. Following treatment, the patient was at castration testosterone level $(<50 \mathrm{ng} / \mathrm{dl})$ persistently, and his PSA levels reduced to a minimum of $0.013 \mathrm{ng} / \mathrm{ml}$.

However, 3 months later, increased levels of PSA were measured, reaching a maximum of $10.73 \mathrm{ng} / \mathrm{ml}$ (similar to the values presented prior to chemotherapy), and the patient also suffered from bone pain. The PET/CT and MRI reevaluation revealed metastases in T4-12, L1-5 and S1-2 vertebral bodies (Figs. 1A, 2A, 3A and 4A), indicating disease progression.

The patient was then suggested the possibility of receiving second-line hormone therapy for HRPC. However, the patient refused this option, and in consequence, was then treated every 3 weeks with docetaxel $\left(75 \mathrm{mg} / \mathrm{m}^{2}\right.$, day 1$)$ and prednisone (5 mg bid, days 1-21). The patient also received bilateral orchiectomy of his own accord, and discontinued the combined androgen blockade therapy following the second cycle of therapy. Since the patient suffered severe neutropenia during this period, granulocyte colony-stimulating factor (G-CSF) was used as an adjunct to the systematical therapy following every cycle of chemotherapy. Additionally, moderate anemia (hemoglobin, $78 \mathrm{~g} / \mathrm{l}$ ) was observed subsequently to the second cycle of chemotherapy; thus, erythropoietin was used during this interval.

Tumor re-evaluation was performed prior to the administration of the third cycle of chemotherapy. The MRI revealed diffuse abnormal signal in almost all the vertebral bodies on T1-, T2-weighted and short TI inversion recovery (STIR) sequence images (Figs. 1B, 2B and 3B). Concomitantly, bone pain relieved, and the PSA levels reduced $>50 \%$, to $3.54 \mathrm{ng} / \mathrm{ml}$. 

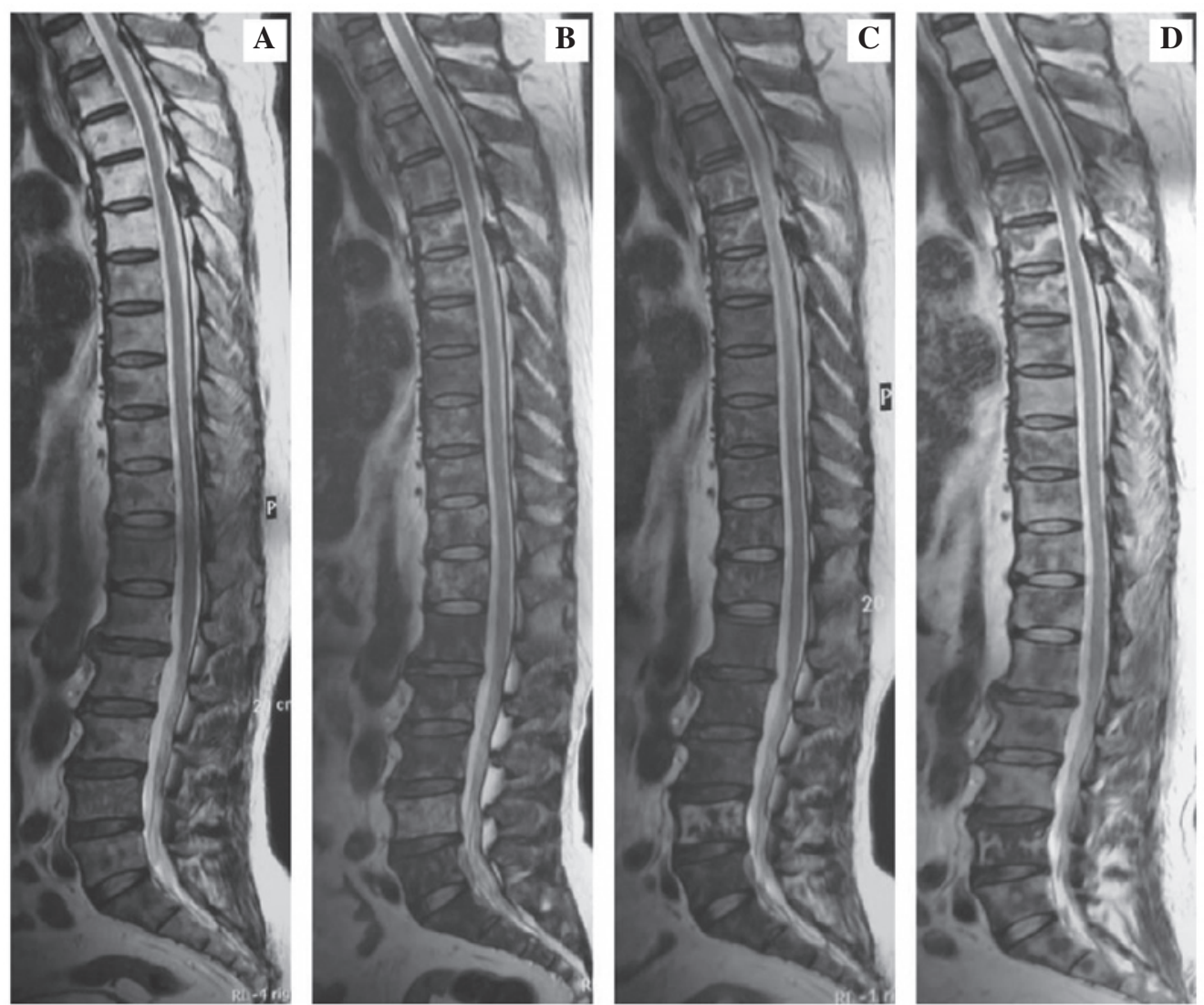

Figure 2. Signal variations observed on MRI T2-weighted images (A) prior to chemotherapy, and following (B) 2, (C) 6 and (D) 10 cycles of chemotherapy treatment. MRI, magnetic resonance imaging.
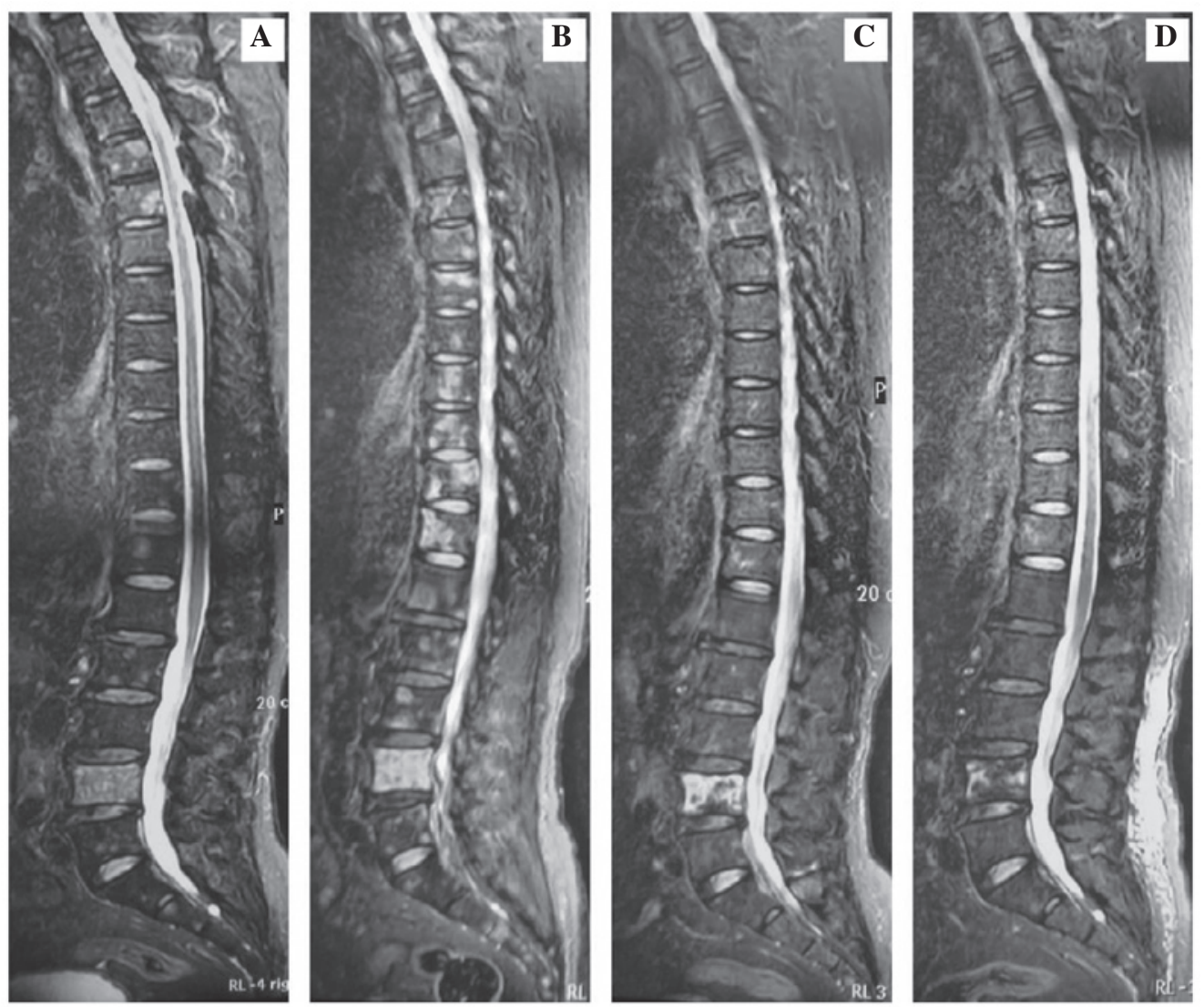

Figure 3. Signal variations observed on MRI STIR-weighted images (A) prior to chemotherapy, and following (B) 2, (C) 6 and (D) 10 cycles of chemotherapy treatment. MRI, magnetic resonance imaging; STIR, short TI inversion recovery. 

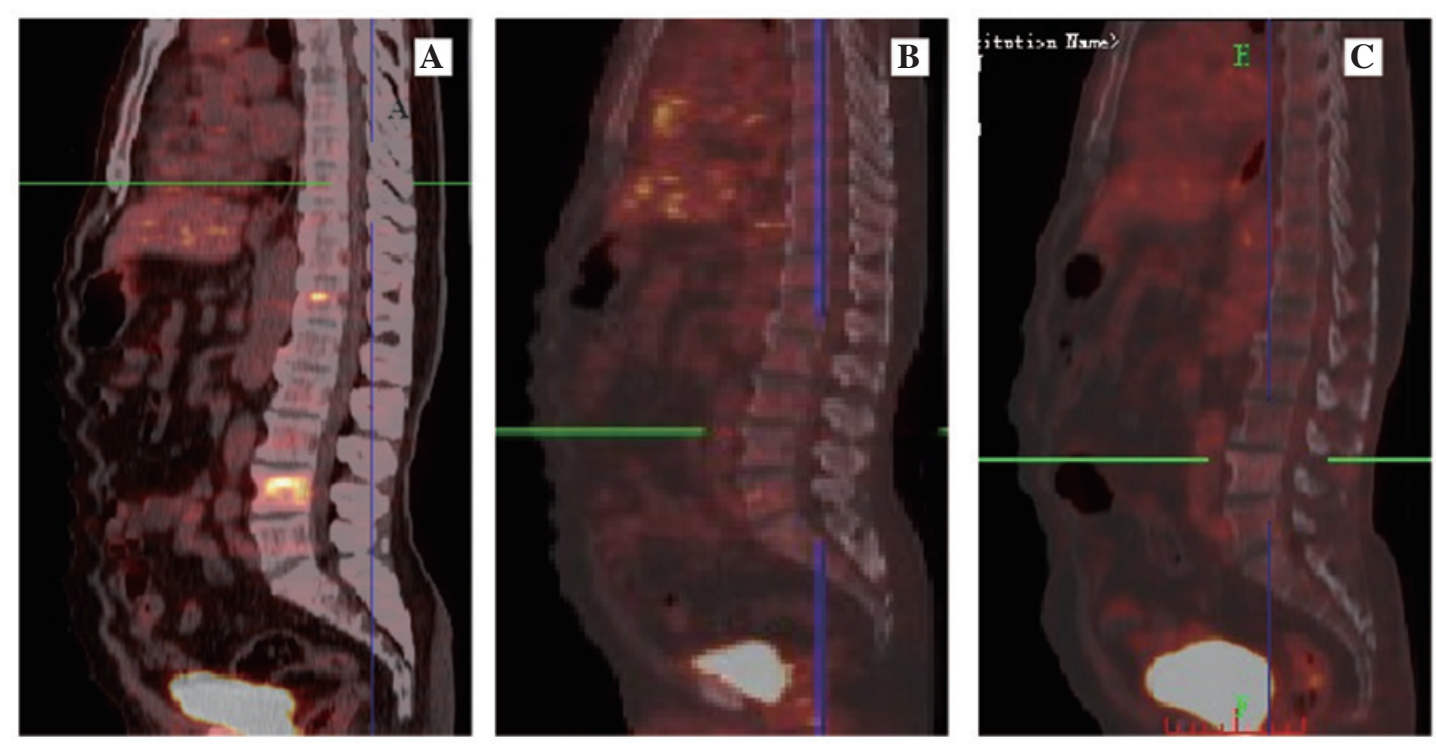

Figure 4. Metastatic transformations observed on PET/CT (A) prior to chemotherapy, and following (B) 6 and (C) 10 cycles of chemotherapy 1 year later. PET, positron emission tomography; CT, computed tomography.

These parameters were combined to assess the therapeutic response. Since the treatment was beneficial to the patient, it was continued, but the dose of docetaxel was reduced to $60 \mathrm{mg} / \mathrm{m}^{2}$ due to the severe neutropenia, and the use of G-CSF as an adjunct following each cycle of the chemotherapy was maintained. At the second re-evaluation following 6 cycles of chemotherapy, the bone lesions on PET/CT had apparently improved (Fig. 4B), compared with the PET-CT results prior to the chemotherapy. The MRI examinations also revealed diffuse signal abnormality (Fig. 1C), which was stable, compared to the T1-weighted images prior to chemotherapy (Fig. 1A); diffuse signal abnormality on T2-weighted images (Fig. 2C); and focal signal abnormality on STIR sequence images (Fig. 3C), demonstrating a marked improvement, compared to the images captured prior to the treatment with chemotherapy (Figs. 1A and 3A).

Based on results derived from the TAX327 study $(8,9)$, the chemotherapy was discontinued following the tenth cycle. Subsequently, MRI was conducted, which revealed focal abnormal signal (Figs. 1D, 2D and 3D), indicating an improvement, compared to the MRI images captured prior to the chemotherapy treatment (Figs. 1A, 2A and 3A).

Zoledronic acid was then administered to the patient intermittently every 3-4 weeks, and 1 year later, the follow-up PET/CT revealed no abnormality on the previous bone metastatic position (Fig. 4C). Currently, the patient is alive without any osseous symptoms.

\section{Discussion}

The accurate assessment of treatment response regarding prostate cancer with bone metastases is crucial. However, unexpected findings on clinical images may occasionally be encountered, which may complicate the diagnosis when the radiologists attempt to interpret these examinations. The present article reviewed the pitfalls of various images aimed to assess the treatment response in patients with prostate cancer presenting with bone metastases, in the context of their mechanisms, and explores how to recognize the false positive images from the true positive ones, in order to accurately assess the therapeutic response.

'Flare' phenomenon. Since the 'flare' phenomenon was first observed in 1972 (10), other studies reporting similar observations have emerged $(4,5)$. The 'flare' phenomenon was defined as an early successful treatment of patients with bone metastases that may be accompanied by an initial apparent deterioration of certain lesions or the appearance of novel lesions on the clinical images, followed by improvement (6). This phenomenon is frequently observed in patients with breast and prostate cancer with bone metastases, following systematic therapy such as endocrine therapy and chemotherapy. It is important to highlight the 'flare' phenomenon in order to avoid a false decision on the basis of a potentially erroneous interpretation of the images in clinical practice.

The 'flare' phenomenon would usually emerge between 2 weeks and 3 months subsequent to the initiation of the efficacious therapy, with reported frequencies of $6-25 \%$ in patients with prostate cancer metastases, and 33\% in patients with treated breast metastases (11). Thus, consensus criterion such as that provided by the Prostate Cancer Working Group 2 (12) indicates that disease progression of bone metastatic patients requires a confirmatory scan that reveals additional lesions, compared to the first follow-up scan, which must be performed $\geq 6$ weeks later, whereas the first follow-up scan is not recommended to be conducted until 12 weeks since the initial date of the treatment, due to the 'flare' phenomenon (12).

Conventional radiography, BS and CT rely on the activation of bone cells (osteoblasts and osteoclast) to detect modifications in the bone trabeculae as a result of neoplastic lesions (13). A possible mechanism for the 'flare' phenomenon is the osteoblastic healing of the bone metastases (14), which has been demonstrated by Messiou et al (11) and Hashisako et al (15). This mechanism may also explain the 'flare' phenomenon on CT, which is capable of differentiating osteoblastic alterations by itself (5). Another mechanism proposed 
by Cook et al (16) suggests that the 'flare' phenomenon would amplify the signal and improve the sensitivity and specificity to detect the occult lesions existing prior to the initiation of the treatment. In their studies, the bones of patients thought not to suffer of bone metastasis on BS were demonstrated to be affected, following an efficacious treatment (16). This possible mechanism of 'flare' phenomenon may be explained by the fact that the occult lesions need time to become visible on $\mathrm{BS}$ and $\mathrm{CT}$ images. In this regard, the prognostic significance of the 'flare' phenomenon must be considered, since certain undetected lesions, which may have been present prior to the treatment, may respond to the treatment. Janicek et al (17) highlighted that the 'flare' phenomenon on BS is a favorable response to therapy not associated with overall survival. Nonetheless, future studies are required to evaluate the prognostic significance of the 'flare' phenomenon.

Marrow reconversion on MRI. MRI is sensitive to the early modifications in bone marrow that precede the osteoclastic/osteoblastic response of the bone matrix to tumor infiltration, prior to bone trabeculae or cortices being affected by the disease (18). A prospective study has determined the sensitivity and specificity to detect the metastatic lesions to be 100 and $88 \%$ for MRI, and 46 and 32\% for BS, respectively (18). Thus, MRI has become a superior tool than BS and $\mathrm{CT}$ for the detection and characterization of numerous neoplastic lesions involving the skeleton.

However, on MRI, marrow reconversion would mimic malignancy, since the malignancy and the red marrow exhibit similar signal variations on MRI (19). There are two main types of bone marrow, red and yellow. Yellow marrow is mainly composed of fat cells with few hematopoietic cells, while red marrow is mainly composed of hematopoietic cells. Yellow marrow appears hyperintense on T1-weighted imaging, and hypointense on T2-weighted imaging, whereas red marrow exhibits an intermediate signal intensity on T1- and T2-weighted images, and exhibits a T1 signal of relatively lower intensity, compared to yellow marrow. On STIR, red marrow displays an intermediate signal that is more intense than fatty marrow and subcutaneous fat, and similar in signal intensity to muscle (20). Bone metastases are hypointense on T1-weighted images due to their high sensitivity in detecting fatty marrow replacement by neoplastic elements, with a high contrast between the low signal intensity of the lesions and the high signal intensity of the surrounding tissues. In addition, bone metastases usually exhibit T2 and STIR hyperintensity (19). Therefore, it is easy to confound marrow reconversion with bone metastasis on MRI.

As the healthy human skeleton matures, a red-yellow marrow conversion begins in childhood, and is usually completed at 25 years of age (21). Generally, red-yellow marrow conversion proceeds from distal to proximal areas in the limbs. In adults, the largest areas of red marrow remain in the vertebrae, pelvis, ribs and sternum, with visible red marrow in the proximal shafts of the femora and humeri (22). Marrow reconversion refers to the process whereby mature yellow marrow is replaced by infantile hematopoietic marrow when the existing marrow can no longer meet the needs for hematopoiesis (20). Demand for increased hematopoiesis occurs in a number of situations, including i) consumption of marrow-stimulating medications such as G-CSF and erythropoietin; ii) anemia; iii) marrow replacement disorders; iv) high altitudes; v) smoking; and vi) obesity. In patients experiencing marrow reconversion, the sites in which red marrow first appears are those areas that last converted to yellow marrow, and this process then continues in reverse physiologic order (22). Therefore, hematopoietic marrow hyperplasia initially affects the axial skeleton, followed by the appendicular skeleton. Previous reports regarding red marrow reconversion mimicking malignancy on MRI were limited to primary musculoskeletal neoplasm (19).

In the present case report, when reviewing the patient's clinical and radiographic course of disease, it is possible to infer that the false positive pitfall was due to the marrow reconversion. In order to avoid such situations in the future, an adequate acquaintance of history of the current disease is essential. It is generally accepted by radiologists and clinicians that elevated bone marrow uptake on PET is induced by G-CSF therapy, and therefore, ${ }^{18}$ fluorodeoxyglucose-PET examination should be delayed in patients receiving G-CSF (23). However the use of G-CSF is often ignored when MRI is performed on the patient. The patient in the present case report received chemotherapy against HRPC, and when subjected to complete blood count, anemia and neutropenia were revealed, as a result of the endocrine therapy and chemotherapy administered. In addition, erythropoietin and G-CSF were used as adjuvants for the anti-tumor therapy. However, the incidence of bone reconversion increases due to anemia, chemotherapy and marrow-stimulating medications such as G-CSF and erythropoietin (20). Previous studies have reported that the time interval from the last dose of GSF to the follow-up MRI in the case of red marrow reconversion should be 0-42 days (mean, 9 days) (19). By identifying the signal variations on MRI, the pre- and post-GSF images should be evaluated in combination with the history of G-CSF application and the corresponding white blood cells response. The pre-and post-GSF scans should be obtained with parameters matched as closely as possible to facilitate comparison. Furthermore, if the radiologist or clinician is uncertain whether the scans reveal red marrow reconversion or tumor, it would be preferable to reimage the area on the opposed-phase images. Seiderer et al (24) demonstrated that the signal in the opposed-phase images correlates with the fat/water fraction. Since normal red marrow exhibits low signal in opposed-phase images, pathological processes such as neoplastic lesions that lead to an increase of water are indicated by a high intensity signal $(24,25)$. These opposed-phase images proved to be useful in the evaluation of hematopoietic hyperplasia as a result of therapy with G-CSF in healthy blood stem cell donors at low-field strength (26). Additionally, in-and out-of-phase gradient-echo MRI of bone marrow signal intensity abnormalities may aid to predict the likelihood of neoplastic or non-neoplastic lesions (25).

Other parameters for treatment assessment. The present review aims to provide suggestions about the assessment of therapeutic response in prostate cancer with bone metastases by parameters other than imaging, since it is unwise to affirm disease progression solely depending on images. Thus, a potential prognostic factor is required in order to avoid the selection of erroneous treatments. The pitfalls of images may be potentially recognized by the evaluation of the patient symptoms, the levels of the tumor marker PSA and the presence of lesions. 
These parameters may provide useful clinical information to oncologists and aid a wise decision. The Prostate Cancer Working Group 2 defined a PSA partial response as $>50 \%$ decline from baseline, measured twice, 3-4 weeks apart (12). The use of a decline $>50 \%$ from baseline as a response measure was derived in part from prognostic factor analyses that associated the degree of decline with survival (27). The PSA response and the relief of bone pain may aid the recognition of these pitfalls in the clinical images, and in consequence, support the continuation of the chemotherapy treatment. Similarly, cases of PSA flare phenomenon (28) and pain flare phenomenon (29) have been previously reported. Therefore, it has been proposed that the pain and PSA response are associated with survival, but are not adequate to use as surrogate end points, according to the TAX-327 study, which developed a prognostic model and nomogram using baseline clinical variables to predict mortality among males diagnosed with castration resistant prostate cancer $(8,9)$. The guidelines of the Prostate Cancer Working Group 2 also emphasize that disease progression should not be solely defined by PSA levels, pain or bone metastases on BS (12). Therefore, individual parameters, including the presence of lesions, levels of PSA, clinical images and pain response, are often combined together in order to assess the therapeutic response and decide accordingly which is the best treatment for the patient.

In conclusion, in patients affected by castration-resistant prostate cancer, it is difficult to assess the therapeutic response and decide which metrics to use when trying to select the most convenient treatment for the patient and the most suitable time for administration, due to the 'flare' phenomenon observed on clinical images and the process of marrow reconversion exhibited on MRI. Therefore, a better understanding of the pitfalls on images, and a more accurate judgment of the treatment response may aid the selection of the most beneficial treatment for patients with prostate cancer.

\section{References}

1. Bashir MN: Epidemiology of Prostate Cancer. Asian Pac J Cancer Prev 16: 5137-5141, 2015.

2. Cooper CR, Chay CH, Gendernalik JD, Lee HL, Bhatia J, Taichman RS, McCauley LK, Keller ET and Pienta KJ: Stromal factors involved in prostate carcinoma metastasis to bone. Cancer 97 (Suppl): 739-747, 2003.

3. Carlin BI and Andriole GL: The natural history, skeletal complications and management of bone metastases in patients with prostate carcinoma. Cancer 88 (Suppl): 2989-2994, 2000.

4. Pollen JJ, Witztum KF and Ashburn WL: The flare phenomenon on radionuclide bone scan in metastatic prostate cancer. AJR Am J Roentgenol 142: 773-776, 1984.

5. Messiou C, Cook G, Reid AH, Attard G, Dearnaley D, de Bono JS and de Souza NM: The CT flare response of metastatic bone disease in prostate cancer. Acta Radiol 52: 557-561, 2011.

6. Ryan CJ, Shah S, Efstathiou E, Smith MR, Taplin ME, Bubley GJ, Logothetis CJ, Kheoh T, Kilian C, Haqq CM, et al: Phase II study of abiraterone acetate in chemotherapy-naive metastatic castration-resistant prostate cancer displaying bone flare discordant with serologic response. Clin Cancer Res 17: 4854-4861, 2011.

7. Tombal B, Rezazadeh A, Therasse P, Van Cangh PJ, Vande Berg B and Lecouvet FE: Magnetic resonance imaging of the axial skeleton enables objective measurement of tumor response on prostate cancer bone metastases. Prostate 65: 178-187, 2005.

8. Berthold DR, Pond G, De Wit R, Eisenberger MA and Tannock IF: Association of pain and quality of life (QOL) response with PSA response and survival of patients (pts) with metastatic hormone refractory prostate cancer (mHRPC) treated with docetaxel or mitoxantrone in the TAX-327 study. J Clin Oncol 24: 4516, 2006.
9. Berthold DR, Pond GR, de Wit R, Eisenberger MA and Tannock IF; TAX 327 Investigators: Survival and PSA response of patients in the TAX 327 study who crossed over to receive docetaxel after mitoxantrone or vice versa. Ann Oncol 19: 1749-1753, 2008.

10. Greenburg EJ, Chu FC, Dwyer AJ, Ziminski EM, Dimich AB and Laughlin JS: Effects of radiation therapy on bone lesions as measured by $47 \mathrm{Ca}$ and $85 \mathrm{Sr}$ local kinetics. J Nucl Med 13: 747-751, 1972.

11. Messiou C, Cook G and de Souza NM: Imaging metastatic bone disease from carcinoma of the prostate. Br J Cancer 101: 1225-1232, 2009.

12. Scher HI, Halabi S, Tannock I, Morris M, Sternberg CN, Carducci MA, Eisenberger MA, Higano C, Bubley GJ, Dreicer R et al; Prostate Cancer Clinical Trials Working Group: Design and end points of clinical trials for patients with progressive prostate cancer and castrate levels of testosterone: Recommendations of the Prostate Cancer Clinical Trials Working Group. J Clin Oncol 26: 1148-1159, 2008.

13. Hamaoka T, Madewell JE, Podoloff DA, Hortobagyi GN, Ueno NT: Bone imaging in metastatic breast cancer. J Clin Oncol 22: 2942-2953, 2004.

14. Pollen JJ and Shlaer WJ: Osteoblastic response to successful treatment of metastatic cancer of the prostate. AJR Am J Roentgenol 132: 927-931, 1979.

15. Hashisako M, Wakamatsu K, Ikegame S, Kumazoe H, Nagata N and Kajiki A: Flare phenomenon following gefitinib treatment of lung adenocarcinoma with bone metastasis. J Exp Med 228: $163-168,2012$

16. Cook GJ, Venkitaraman R, Sohaib AS, Lewington VJ, Chua SC, Huddart RA, Parker CC, Dearnaley DD and Horwich A. The diagnostic utility of the flare phenomenon on bone scintigraphy in staging prostate cancer. Eur J Nucl Med Mol Imaging 38: 7-13, 2011.

17. Janicek MJ, Hayes DF and Kaplan WD: Healing flare in skeletal metastases from breast cancer. Radiology 192: 201-204, 1994.

18. Lecouvet FE, Geukens D, Stainier A, Jamar F, Jamart J, d'Othée BJ, Therasse P, Vande Berg B and Tombal B: Magnetic resonance imaging of the axial skeleton for detecting bone metastases in patients with high-risk prostate cancer: Diagnostic and cost-effectiveness and comparison with current detection strategies. J Clin Oncol 25: 3281-3287, 2007:

19. Hartman RP, Sundaram M, Okuno SH and Sim FH: Effect of granulocyte-stimulating factors on marrow of adult patients with musculoskeletal malignancies: Incidence and MRI findings. AJR Am J Roentgenol 183: 645-653, 2004.

20. Long SS, Yablon CM and Eisenberg RL: Bone marrow signal alteration in the spine and sacrum. AJR Am J Roentgenol 195: W178-W200, 2010.

21. Vogler JB III and Murphy WA: Bone marrow imaging. Radiology 68: 679-693, 1988.

22. Kricun ME: Red-yellow marrow conversion: Its effect on the location of some solitary bone le-sions. Skeletal Radiol 14: 10-19, 1985.

23. Johnston KL, Farnen JP, Manske BR and Go RS: Abnormal positron emission tomography (PET) scan secondary to the use of hematopoietic growth factors. Haematologica 90: EIM03, 2005.

24. Seiderer M, Staebler A and Wagner H: MRI of bone marrow: Opposed-phase gradient-echo sequences with long repetition time. Eur Radiol 9: 652-661, 1999.

25. Disler DG, McCauley TR, Ratner LM, Kesack CD and Cooper JA: In-phase and out-of-phase MR imaging of bone marrow: Prediction of neoplasia based on the detection of coexistent fat and water. AJR Am J Roentgenol 69: 1439-1447, 1997.

26. Altehoefer C, Bertz H, Ghanem NA and Langer M: Extent and time course of morphological changes of bone marrow induced by granulocyte-colony stimulating factor as assessed by magnetic resonance imaging of healthy blood stem cell donors. J Magn Reson Imaging 14: 141-145, 2001.

27. Scher HI, Kelly WM, Zhang ZF, Ouyang P, Sun M, Schwartz M, Ding C, Wang W, Horak ID and Kremer AB: Post-therapy serum prostate-specific antigen level and survival in patients with androgen-independent prostate cancer. J Natl Cancer Inst 91: 244-251, 1999.

28. Nelius T, Klatte T, de Riese W and Filleur S: Impact of PSA flare-up in patients with hormone-refractory prostate cancer undergoing chemotherapy. Int Urol Nephrol 40: 97-104, 2008.

29. Sartor O, Reid RH, Hoskin PJ, Quick DP, Ell PJ, Coleman RE, Kotler JA, Freeman LM and Olivier P; Quadramet 424Sm10/11 Study Group: Samarium-153-Lexidronam complex for treatment of painful bone metastases in hormone-refractory prostate cancer. Urology 63: 940-945, 2004. 